\title{
Conductance fluctuations as a tool for investigating the quantum modes in atomic-size metallic contacts
}

\author{
B. Ludoph and J. M van Ruitenbeek \\ Kamerlingh Onnes Laboratorium, Universiteit Leiden, Postbus 9504, 2300 RA Leiden, The Netherlands
}

(Received 6 July 1999)

\begin{abstract}
Recently it has been observed that the conductance fluctuations of atomic-size gold contacts are suppressed when the conductance is equal to an integer multiple of the conductance quantum. The fact that these contacts tend to consist exclusively of fully open or closed modes has been argued to be the origin for this suppression. Here the experiments have been extended to a wide range of metallic elements with different chemical valences, and they provide information about the relation between the mode composition and statistically preferred conductance values observed in conductance histograms.
\end{abstract}

\section{INTRODUCTION}

Manipulation and characterization of atoms and atomicsize metallic constrictions has recently become available through the development of the scanning tunneling microscope. ${ }^{1}$ An alternative tool, for creating stable and clean atomic size metallic contacts, is the mechanically controllable break junction (MCB)., ${ }^{2,3}$ For a characterization of these systems, measurements of the electrical conductance are widely employed. This is a result of the ease with which they usually can be obtained. The framework within which one should describe the conductance of such small contacts, which in the case of metals have dimensions on the order of the Fermi wavelength, is the Landauer-Büttiker formalism. ${ }^{4}$ In this formalism the conductance in the contact is described by $N$ channels, determined by the narrowest cross section of the constriction and the Fermi wavelength. Each channel has a transmission probability $T_{n}$ with a value between 0 and 1 . The total conductance is given by $G=\left(2 e^{2} / h\right) \sum_{n=1}^{N} T_{n}$. For an adiabatic constriction in a free-electron gas, the conductance increases stepwise with quantum units of the conductance $\left(G_{0}=2 e^{2} / h\right)$ as the channels open one by one while increasing the constriction diameter. ${ }^{5}$ However, when one pulls apart a metallic atomic-size contact, neither the diameter nor the conductance of the constriction decreases smoothly. Instead, a series of steps (of order $G_{0}$ ) and plateaus are observed in the conductance on elongation of the contact. The sequence of steps and plateaus is different each time the contact is pulled apart. The steps correspond to atomic reconfigurations, and the plateaus to elastic deformation of the contact. ${ }^{6}$ It is tempting, but in principle incorrect, to assume offhand that these conductance measurements on atomic necks simply probe a series of discrete diameters of a free-electron gas. It may work for some metals (we will show that for sodium this is nearly the case), but a correct general description of the conductance of metallic point contacts consisting of (even in the simplest case) a single atom has to consider the chemical valence of this atom. ${ }^{7,8} \mathrm{Com}-$ pare, for instance, a single-atom contact of the monovalent $s$ metal gold with the trivalent $s p$ metal aluminum. In the former case the conductance is carried by a single channel with conductance close to $G_{0}$. A single atom of aluminum, on the other hand, with three conduction electrons in the $3 s$ and $3 p$ shells, also has a total conductance close to $G_{0}$ but allows three partially transmitting channels.

Recently ${ }^{9}$ a technique, making use of conductance fluctuations, has been presented which does not require superconductivity ${ }^{8,10}$ to obtain information about the conductance modes contributing to the conductance. First results on gold contacts with a conductance up to $5 G_{0}$ have shown that, for this $s$ metal, once a channel (with number $n$ ) is partially open it tends to fully open before a next $(n+1)$ th channel starts to contribute significantly. This interpretation was confirmed by an independent technique which consists of measuring the shot noise in the point-contact current. ${ }^{11}$ In this paper we present a more complete argumentation of the theory, together with measurements of the conductance fluctuations on copper, silver, sodium, aluminum, niobium, and iron, and discuss what information on the channel transmissions can be extracted from our results. We will also compare our conductance fluctuation results to recently published measurements on the thermopower of atomic size contacts, and show that both measurements can be related without any free parameters.

Fluctuations in the conductance with bias voltage have previously been observed in larger ballistic contacts, ${ }^{12}$ and have an origin analogous to the universal conductance fluctuations measured in diffusive wires. ${ }^{13}$ The interesting aspect of such fluctuations in quantum point contacts is that their rms amplitude depends on the transmission probability of the channels contributing to the conductance. The underlying principle of this effect can be understood by considering a contact with a single conducting mode having a finite transmission probability $T$, described by transmission and reflection coefficients $t, t^{\prime}, r$, and $r^{\prime}$ (coming in from left and right, respectively), with $\left|t^{\prime}\right|^{2}=|t|^{2}=T$ and $\left|r^{\prime}\right|^{2}=|r|^{2}=$ $1-T$. As illustrated in Fig. 1, electron waves transmitted by the contact with amplitude $t$, and scattered back toward the contact through diffusive paths in the bank with probability amplitude $a$, have a probability amplitude $r$ to be reflected at the contact. This wave interferes with the directly transmitted partial wave and modifies the total conductance, depending on whether the resulting interference is constructive or destructive. A similar contribution comes from the trajectories 


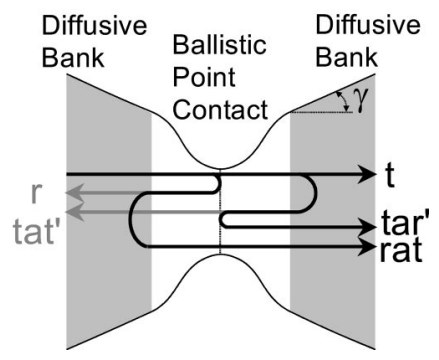

FIG. 1. Schematic diagram of the configuration used in the theoretical analysis. The dark lines with arrows show the paths, which interfere with each other and contribute to the conductance fluctuations in lowest order.

on the other side of the contact. The interference terms will be sensitive to changes in the phase accumulated along the trajectories, which is determined by the electron energy and the path length. The fluctuations in the conductance as a function of bias voltage are thus the result of the change of these phase factors by the increase in the kinetic energy of the electrons by an amount $e V$. What is immediately apparent from the principle illustrated in Fig. 1 is that when the coefficient $T$ is either 0 or 1 , the interference and thus the amplitude of the fluctuations vanishes. This suppression of conductance fluctuations at quantized values has been noted in numerical simulations of quantum point contacts containing disorder by Maslov et al. ${ }^{14}$

Each time the contact is opened and closed again to sufficiently large conductance values, random atomic reconfigurations take place, leading to a different set of scattering centers. The statistical results of many different contacts can hence be interpreted as the ensemble average over defect configurations. With this technique we have studied the average properties of the conductance modes for different materials, and their relation to the statistically preferred conductance values observed previously by various authors ${ }^{15-20}$ through the measurement of so-called conductance histograms.

Typically when studying conductance fluctuations, one measures the differential conductance over a wide range of bias voltage or magnetic field. Here, on the other hand, we measure the first and second derivatives of the current with respect to voltage of atomic-size contacts. The first derivative gives us the conductance, and the second derivative is a parametric derivative of the conductance. The latter can roughly be seen as a measure for the amplitude of the fluctuations with voltage. We measure these quantities for a large number of different contacts, thus effectively determining an average over an ensemble of scattering configurations. This measurement method is preferable, as it is much faster than measuring the conductance as a function of bias voltage directly, and hence allows the experimental determination of the average properties of many contacts within a reasonable time scale.

\section{THEORY}

In this section we will give a more detailed description of the phenomenological theory presented in Ref. 9. In our model for a metallic constriction we divide the conductor into three separate regions: First is a region, small on the scale of the scattering lengths involved, centered around the narrowest part of the conductor, which we describe as ballistic. On either side of this ballistic region we consider a diffusive region, characterized by a mean free path $l_{e}$. In order to make the geometry of the contact more realistic, we assume a conical shape for these diffusive regions, with opening angle $\gamma$ (see Fig. 1). The probability amplitudes for scattering from any incoming mode on the left to any outgoing mode on the right side of this ballistic section (henceforth referred to as the "bare contact") is described in terms of the matrices of transmission $\mathbf{t}$ and $\mathbf{t}^{\prime}$, and reflection, $\mathbf{r}$ and $\mathbf{r}^{\prime}$, when coming from the left and right respectively:

$$
\left(\begin{array}{c}
o_{r} \\
i_{r}
\end{array}\right)=\mathbf{M} \times\left(\begin{array}{c}
i_{l} \\
o_{l}
\end{array}\right)=\left(\begin{array}{lll}
\mathbf{t}^{\dagger-1} & \mathbf{r}^{\prime} \mathbf{t}^{-1} \\
-\mathbf{t}^{\prime-1} & \mathbf{r} & \mathbf{t}^{\prime-1}
\end{array}\right)\left(\begin{array}{c}
i_{l} \\
o_{l}
\end{array}\right),
$$

where $\mathbf{M}$ is the transfer matrix and $i_{r}, o_{r}, i_{l}$, and $o_{l}$ are the vectors of the incoming and outgoing waves for the rightand left-hand sides, respectively. The matrix of transmission probabilities is given by $\mathbf{T}=\mathbf{t t}^{\dagger}$, which can be diagonalized. ${ }^{21,22}$ For a narrow constriction, most of the diagonal elements will be zero. The number of conducting modes, $N$, and their transmission probabilities are given by the nonzero diagonal elements $T_{n}=\left|t_{n}^{2}\right|=\left|t_{n}^{\prime 2}\right| \quad n$ $=1,2, \ldots, N$. The reflection probability of mode $n$ is given by $R_{n}=\left|r_{n}^{2}\right|=\left|r_{n}^{\prime 2}\right|=1-T_{n}$. In the simple free-electron-gas model, the number $N$ is determined by the width of the narrowest part of the contact ${ }^{4}$ and by the Fermi wavelength. Note, however, that in principle it is not restricted to any particular model. The values of the $T_{n}$ 's are somewhat influenced by our arbitrary choice of the boundaries between the ballistic and diffusive regions. For this influence to be small, the distance $L$ between the center of the contact and the boundaries should be large on the scale of the contact diameter. On the other hand, in order to be able to neglect fluctuations of $T_{n}$ on the scale of the applied voltage, $V$, we require $L \ll \hbar \mathrm{v}_{F} / e V$. For metallic contacts we can typically take $L \simeq 1 \mathrm{~nm}$.

The left and right banks are also described in terms of transfer matrices $\mathbf{M}_{l, r}$, similar in form to the one used for the bare contact in Eq. (1). In this case we define $\mathbf{t}_{r}$ and $\mathbf{a}_{r}$ as the transmission matrix and return amplitude matrix for the right bank, and $\mathbf{t}_{l}$ and $\mathbf{a}_{l}$ the corresponding ones for the left bank. The elements of the return amplitude matrices $a_{l_{m n}}$ and $a_{r_{m n}}$, which scatter a wave from mode $m$ to mode $n$ on the left- or right-hand side diffusive sections, are expected to be small compared to 1 . It is, hence, a reasonable approximation to calculate the total transmission probability to first order in the return amplitude matrix elements only. The return amplitudes are energy dependent, but this will not be made explicit until this dependence becomes relevant in Eq. (5). The total transmission matrix for the two banks and ballistic constriction combined can be written as

$$
\begin{aligned}
\mathbf{t}_{t} & =\left[\left(\mathbf{M}_{r} \mathbf{M} \mathbf{M}_{l}\right)_{22}\right]^{-1} \\
& =\mathbf{t}_{l}^{\prime}\left(\mathbf{t}^{\prime-1}-\mathbf{a}_{r} \mathbf{t}^{\dagger-1} \mathbf{a}_{l}-\mathbf{t}^{\prime-1} \quad \mathbf{r a}_{l}-\mathbf{a}_{r} \mathbf{r}^{\prime} \mathbf{t}^{\prime-1}\right)^{-1} \mathbf{t}_{r}^{\prime} .
\end{aligned}
$$

Since the return amplitudes will usually be small we can set $\mathbf{t}_{l}=\mathbf{t}_{r} \simeq \mathbf{I}$, the identity matrix. With this assumption and the fact that we are only calculating to lowest order in $a_{l, r_{m n}}$ we 
are neglecting corrections to the total conductance and higher-order contributions to the conductance fluctuations. These corrections will be discussed at the end of this section.

In order to calculate the conductance fluctuations, we will make an expansion of the total transmission probability to lowest order in $a_{l, r_{m n}}$. Using $\mathbf{t}_{t} \mathbf{t}_{t}^{\dagger}=\left[\left(\mathbf{t}_{t}^{-1}\right)^{\dagger}\left(\mathbf{t}_{t}^{-1}\right)\right]^{-1}$ and assuming the matrices $\mathbf{t}, \mathbf{t}^{\prime}, \mathbf{r}$, and $\mathbf{r}^{\prime}$ are already in diagonal form, the trace of $\mathbf{t}_{t} \mathbf{t}_{t}^{\dagger}$ to lowest order in $a_{l, r_{m n}}$ can be approximated by the sum of the inverse of the diagonal components of $\left(\mathbf{t}_{t}^{-1}\right)^{\dagger}\left(\mathbf{t}_{t}^{-1}\right)$ :

$$
\begin{aligned}
\operatorname{Tr}\left[\mathbf{t}_{t} \mathbf{t}_{t}^{\dagger}\right] & \approx \sum_{n=1}^{N}\left(\frac{1}{T_{n}^{-1}-T_{n}^{-1} 2 \operatorname{Re}\left(a_{r_{n n}} r_{n}^{\prime}+r_{n} a_{l_{n n}}\right)}\right) \\
& \approx \sum_{n=1}^{N} T_{n}\left[1+2 \operatorname{Re}\left(a_{r_{n n}} r_{n}^{\prime}+r_{n} a_{l_{n n}}\right)\right]
\end{aligned}
$$

We assume that the Boltzmann constant times the temperature, $k_{B} \theta$, is much smaller than the energy scale of the applied voltage $e V$ (in accordance with the situation in our experiment), so that we can take the zero temperature approximation. The current is then determined by Eq. (3) through $^{4}$

$$
I=\frac{2 e^{2}}{h} \int_{0}^{e V} \operatorname{Tr}\left[\mathbf{t}_{t} \mathbf{t}_{t}^{\dagger}\right] d E .
$$

The fluctuations in the conductance are described by $\delta G$ $=G-\langle G\rangle$, with $G=\partial I / \partial V$. Combining this with Eqs. (3) and (4), and using $\langle G\rangle=\left(2 e^{2} / h\right) \sum_{n=1}^{N} T_{n}$ to first order in $a_{l, r_{m n}}$, leads to

$$
\begin{aligned}
\delta G(V)= & \sum_{n=1}^{N} \frac{\partial}{\partial e V} \int_{0}^{e V 2} \frac{e^{2}}{h} T_{n} 2 \operatorname{Re}\left[r_{n} a_{l_{n n}}(e V-E)\right. \\
& \left.+a_{r_{n n}}(-E) r_{n}^{\prime}\right] d E \\
= & -\sum_{n=1}^{N} \frac{2 e^{2}}{h} T_{n} 2 \operatorname{Re}\left[r_{n}^{\prime} a_{r_{n n}}(e V)-r_{n} a_{l_{n n}}(-e V)\right] .
\end{aligned}
$$

The return amplitudes contain random phase factors of the form $\exp \left[-i\left(E_{F} \pm e V\right) \tau / \hbar\right]$, where $\tau$ is the traversal time for a particular trajectory. Averaging over the ensemble of defect configurations will give a zero result, $\langle\delta G\rangle=0$. For the correlation function of the conductance as a function of voltage, however, we obtain a finite contribution. In the product, only terms of the form $a_{l, r_{n n}}\left(E_{1}\right) a_{l, r_{n n}}^{*}\left(E_{2}\right)$ have a chance to survive the averaging. In addition, diffusion in the left and right banks is uncorrelated, so that products of $a_{l_{n n}}\left(E_{1}\right)$ and $a_{r_{n n}}\left(E_{2}\right)$ average to zero:

$$
\left\langle\delta G\left(e V_{1}\right) \delta G\left(e V_{2}\right)\right\rangle=\sum_{n=1}^{N}\left(\frac{2 e^{2}}{h} T_{n}\right)^{2} R_{n}\left\langle 2 \operatorname{Re}\left[a_{r_{n n}}\left(e V_{1}\right) a_{r_{n n}}^{*}\left(e V_{2}\right)+a_{l_{n n}}\left(-e V_{1}\right) a_{l_{n n}}^{*}\left(-e V_{2}\right)\right]\right\rangle
$$

At this point we assume that the average properties of the scattering on both sides of the contact, for all the mode indexes, are the same. Further, we propose that $\left\langle a_{l, r_{n n}}\left(E_{1}\right) a_{l, r_{n n}}^{*}\left(E_{2}\right)\right\rangle$ can be expressed as $\int_{0}^{\infty} P_{c l}(\tau) e^{-i\left(E_{1}-E_{2}\right) \tau / \hbar} d \tau$, where $\tau$ is the time required for the completion of a classical diffusive trajectory, and $P_{c l}(\tau)$ is the classical probability distribution to return to the contact at this time. The classical return probability $P_{c l}(\tau)$ can be obtained by considering an electron being injected from the ballistic central section of the contact into the diffusive region at the left or right. When we take the interface between the ballistic and diffusive regions to be at a small distance $L$ from the contact center, then after a given time $\tau$, with $D \tau$ $\gg L^{2}$, the probability distribution to find the electron at a distance $r>L$ is given by the classical result

$$
\rho(\tau, r) d \tau=\frac{2}{(1-\cos \gamma)(4 \pi D \tau)^{3 / 2}} e^{(r-L)^{2} / 4 D \tau} d \tau
$$

where $D=\mathrm{v}_{F} l_{e} / 3$ is the diffusion constant, with $l_{e}$ the mean free path for elastic scattering. Here we have assumed that the diffusive region has the shape of a cone with opening angle $\gamma$ (Fig. 1), and that only a small number of channels are transmitting, so that most electrons entering the ballistic region are reflected. The probability per unit time to find the particle back in a disk of radius $\sigma$ and thickness $d x$ at the entrance of the ballistic region is $\rho(\tau, L) \pi \sigma^{2} d x$. The average time the particle spends in this volume is $d x /\left\langle\mathrm{v}_{x}\right\rangle$ $=\sqrt{3} d x / \mathrm{v}_{F}$. The probability that the particle moves toward the contact instead of away from it is $1 / 2$, and we assume that it has an equal probability to enter into any of the $N_{b}$ modes available at the entrance of the ballistic section, where $N_{b}=\left(k_{F} \sigma / 2\right)^{2}$. Thus the probability per unit time to return to the contact after a time $\tau$, into a given mode, is

$$
P_{c l}(\tau)=\frac{\mathrm{v}_{F}}{2 \sqrt{3 \pi} k_{F}^{2}(D \tau)^{3 / 2}(1-\cos \gamma)} .
$$

This distribution should further be multiplied with a factor $e^{\tau / \tau_{\phi}}$ in order to take into account the probability that within 
a typical time $\tau_{\phi}$ the electron undergoes scattering which destroys phase memory. Combining these expressions we obtain

$$
\begin{aligned}
\left\langle\delta G\left(V_{1}\right) \delta G\left(V_{2}\right)\right\rangle & \\
= & \sum_{n=0}^{N} 4\left(\frac{2 e^{2}}{h} T_{n}\right)^{2}\left(1-T_{n}\right) \int_{0}^{\infty} P_{c l}(\tau) \\
& \times \cos \frac{e\left(V_{1}-V_{2}\right) \tau}{\hbar} e^{-\tau / \tau_{\phi}} d \tau .
\end{aligned}
$$

In our measurements we really measure $\left\langle(\partial G / \partial V)^{2}\right\rangle$ with a fixed modulation voltage rather than $\left\langle\delta G\left(e V_{1}\right) \delta G\left(e V_{2}\right)\right\rangle$. This can easily be corrected by differentiating Eq. (7) with respect to $V_{1}$ and $V_{2}$, and then setting $V=V_{1}=V_{2}$. In the limit of $V \rightarrow 0$, and using the above approximation for the average return probability we obtain

$$
\begin{aligned}
\sigma_{G V}^{2} \equiv & \left\langle\left(\frac{\partial \delta G}{\partial V}\right)^{2}\right\rangle \\
= & G_{0}^{2} \sum_{n=0}^{N} T_{n}^{2}\left(1-T_{n}\right) \frac{4 e^{2} \mathrm{v}_{F}}{\sqrt{3 \pi} \hbar^{2} k_{F}^{2}} \frac{1}{1-\cos \gamma} \frac{1}{D^{3 / 2}} \\
& \times \int_{0}^{\infty} \sqrt{\tau} e^{-\tau / \tau_{\phi}} d \tau .
\end{aligned}
$$

Evaluating the integral and taking the square root results in

$$
\sigma_{G V}=\frac{\sqrt{6} e G_{0}}{\hbar k_{F} \mathrm{v}_{F} \sqrt{1-\cos \gamma}}\left(\frac{\tau_{\phi}}{\tau_{e}}\right)^{3 / 4} \sqrt{\sum_{n=0}^{N} T_{n}^{2}\left(1-T_{n}\right)} .
$$

The typical time scale on which a first collision takes place is the elastic-scattering time $\tau_{e}=l_{e} / \mathrm{v}_{F}$.

We have conducted our experiment by measuring the first and second derivatives of the current with respect to voltage. The amplitude of the applied modulation voltage was responsible for the energy cutoff rather than the dephasing time, as we have assumed above $\left(e V_{\text {mod }}>\hbar / \tau_{\phi}\right)$. Using the derivation in the Appendix, which incorporates this finite modulation voltage into the theory gives us the final result ${ }^{23}$

$$
\sigma_{G V}=\frac{2.71 e G_{0}}{\hbar k_{F} \mathrm{v}_{F} \sqrt{1-\cos } \gamma}\left(\frac{\hbar / \tau_{e}}{e V_{\text {mod }}}\right)^{3 / 4} \sqrt{\sum_{n=1}^{N} T_{n}^{2}\left(1-T_{n}\right)} .
$$

For the fluctuations in the conductance we have described above, terms higher in order than $a_{l, r_{m n}}$, were not very important. However, when all the $N$ channels contributing to the conductance are fully open, the first-order contribution we have calculated above will be zero. Under this condition the second-order terms may have a noticeable contribution. In this case we can take $\mathbf{t}=\mathbf{I}$ and $\mathbf{r}=\mathbf{0}$ in Eq. (2), which greatly simplifies the derivation. It is then quite easy to show that at quantized values $G=N G_{0}$, the contribution of the second-order term in $a_{l, r_{m n}}$ is $\sigma_{G V} \propto N \sqrt{\left\langle\left|a_{l_{m n}}\right|^{2}\left|a_{r_{m n}}\right|^{2}\right\rangle}$. These terms are too small to explain the reduction of the depths of the minima in the experiment discussed below, and will be further ignored.
These higher-order terms, however, are not negligible when we consider the total conductance of the contact. The importance of these higher-order corrections becomes apparent when we compare our theory to the experiments, and notice that these effects result in a significant correction to the total conductance $G$. The necessity to include these conductance corrections in our analysis is a direct consequence of the fact that in the experiment we cannot measure the bare contact conductance alone, as we always measure it in series with the banks. This feature in the conductance of quantum point contacts is usually referred to as the series resistance. $^{15,16,24}$

The lowest-order correction to the average total transmission probability is given by $\left\langle\operatorname{Tr}\left[\mathbf{t}_{t} \mathbf{t}_{t}^{\dagger}\right]\right\rangle=\sum_{n=1}^{N} T_{n}[1$ $\left.-\sum_{m=1}^{N} T_{m}\left(\left\langle\left|a_{l_{m n}}\right|^{2}\right\rangle+\left\langle\left|a_{r_{m n}}\right|^{2}\right\rangle\right)\right]$. The last term describes the path of an electron that is transmitted through the contact, scattered back toward the contact in the diffusive bank, and then transmitted through the contact a second time in the opposite direction. These processes will lead to a smaller conductance than expected for the bare contact conductance alone, since part of the transmitted electrons is scattered back, reducing the net forward current flow.

At higher conductance values, we expect a significant contribution of even higher-order terms in the return probability $a_{l, r_{m n}}$ to the conductance correction, and that hence the lowest-order correction used above will not suffice. Keeping track of higher-order terms becomes very complicated for many channels. However, using random-matrix theory, an expression for the correction to the conductance of a quantum point contact connected to diffusive leads was already derived by Beenakker and Melsen: ${ }^{25}$

$$
\frac{\langle G\rangle}{G_{0}}=\frac{g}{1+(g+1) r}+\frac{1}{3}\left(\frac{(g+1) r}{1+(g+1) r}\right)^{3} .
$$

Here $g=\sum_{n=1}^{N} T_{n}$ is the reduced conductance of the bare contact, where in the theory all channels were assumed to be perfectly transmitting. The diffusive scattering in the banks is represented by $r=G_{0} / G_{d}$, with $G_{d}$ the diffusive conductance of the banks. When one makes the assumption that the conductance of the banks is large compared to the conductance of the contact, Eq. (9) can be simplified and rewritten in a form where the correction to the conductance effectively becomes a somewhat contact-dependent series resistance:

$$
\langle\delta R(g)\rangle \approx\left(1+\frac{1}{g}\right)\left(\frac{1}{G_{d}}\right) .
$$

To lowest order, Eqs. (9) and (10) are consistent with the correction to the average total transmission probability derived from the backscattering above.

\section{EXPERIMENTAL METHOD}

We have used a MCB to make stable atomic-size metallic contacts. The technique ${ }^{2,3}$ uses a notched metal wire glued onto an electrically insulated phosphor bronze bending beam. In the case of sodium a slightly different sample fixation method was required due to its high oxidation rate (see Krans et al. ${ }^{15}$ ). The sample is placed in a three-point bending configuration, which consists of two counter supports and a 
drive at the center of the bending beam powered by a piezoelectric element in combination with a mechanical screw. By first turning the screw and later expanding the piezo by applying a voltage over it, we can bend the substrate in a controlled way, elongating the wire until it finally breaks. The wire is broken at low temperature $(4.2 \mathrm{~K})$ in an evacuated can to ensure that two clean freshly broken surfaces are measured. The voltage applied over the piezoelectric element is linear with the elongation of the contact (for further details, see Ref. 26).

The conductance measurements are performed by applying a $48-\mathrm{kHz}, 20-\mathrm{mV}$ amplitude, sinusoidal voltage over the contact, which is in series with a $100-\Omega$ resistor. The first and second harmonics of the voltage over the resistor are measured, from which we obtain the first $(G=\partial I / \partial V)$ and second $\left(\partial G / \partial V=\partial^{2} I / \partial V^{2}\right)$ derivatives of the current with respect to the voltage of the contact. The conductance is determined with an accuracy better than $1 \%$ for values larger than $0.5 G_{0}$. We use a HP3325b function generator to produce the modulation voltage, while two Stanford Research SR830 lock-in amplifiers at $f$ and $2 f$, with a time constant of $10 \mathrm{~ms}$, are used to obtain the first and second derivative. 16-bit analog-to-digital and digital-to-analog converters are used to control and measure the piezo voltage. A PC-based controller sweeps the piezo voltage up, and while the contact breaks, the readings of $G$ and $\partial G / \partial V$ are taken through an IEEE connection every $100 \mathrm{~ms}$. A full curve of the contact from a conductance of over $20 G_{0}$ to the transition to vacuum tunneling is recorded in about $30 \mathrm{~s}$.

A large number of such curves have been taken for gold, silver, copper, sodium, aluminum, niobium, and iron. In order to avoid anomalously large $\partial G / \partial V$ values due to unstable contacts near a conductance step, and to avoid measuring the average properties of two different plateaus as a result of the finite integration time of the lock-in amplifiers (which average $G$ and $\partial G / \partial V$ over $10 \mathrm{~ms}$ ), only points on a plateau are included through exclusion of data points, with suitable selection criteria, for which the deviation of $G$ and $\partial G / \partial V$ with respect to previous and consecutively recorded data points is too large. After applying this exclusion procedure to each of these materials, we have analyzed the results by combining all the data and sorting them according to conductance. Then a fixed number of consecutive data points were taken from which $\sigma_{G V}=\sqrt{\left\langle(\partial G / \partial V)^{2}\right\rangle-\left(\langle\partial G / \partial V\rangle^{2}\right)}$, and the average conductance value were determined. With this method we obtained $\sigma_{G V}$ as a function of conductance, in a way which is independent of the number of sampled points at a particular conductance value.

\section{NOBLE METALS COPPER, SILVER, AND GOLD}

The three panels in Fig. 2 show the measurement of the differential conductance, obtained with a small $(<0.35 \mathrm{mV})$ modulation voltage, against bias voltage for three gold atomic-size contacts. In each case two curves are plotted, one for increasing bias voltage and one for decreasing bias voltage, showing the reproducibility of the behavior. The bias voltage over the contact determines the energy of the electrons injected into the banks, and hence modifies the electron interference resulting from electrons scattered back toward the contact in the banks. This change in the interfer-

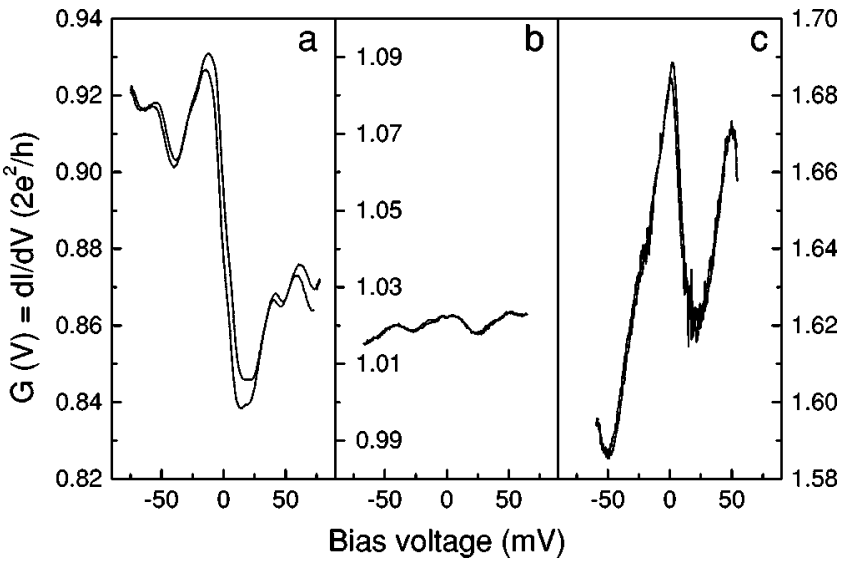

FIG. 2. Plotted in the three panels is the differential conductance $\partial I / \partial V$ as a function of bias voltage, measured with a modulation amplitude $<0.35 \mathrm{mV}$, for three different gold contacts with $G$ (a) $\sim 0.88 G_{0}$, (b) $\sim 1.02 G_{0}$, and (c) $\sim 1.65 G_{0}$. For all three curves the vertical scale spans $0.12 G_{0}$.

ence gives rise to the fluctuations shown in Fig. 2. In our experiments described below the voltage dependence of the conductance is determined with a modulation amplitude of $20 \mathrm{mV}$, i.e., the average slope of curves such as those presented in Fig. 2 is determined over a bias voltage range of $\pm 20 \mathrm{mV}$. Note the small amplitude of the fluctuations in Fig. 2(b). We will argue later that this is an example of the reduction of $\sigma_{G V}$ for the conductance of gold contacts with value near $1 G_{0}$ due to the $\sqrt{T_{n}^{2}\left(1-T_{n}\right)}$ factor in Eq. (8).

An example of the typical conductance and $\partial G / \partial V$ behavior when breaking a gold contact for a constant modulation amplitude of $20 \mathrm{mV}$ and zero bias is presented in Fig. 3 . The steps and plateaus in the conductance correspond with atomic rearrangements and elastic deformation respectively, as the contact is pulled apart and finally breaks. ${ }^{6}$ At each step in the conductance we find a corresponding step in $\partial G / \partial V$. Even tiny steps in the conductance, such as between $7 G_{0}$ and $8 G_{0}$, can produce dramatic jumps in $\partial G / \partial V$. Changes in electron path lengths of the order of the Fermi wavelength (which is the atomic scale for metals) occur at these steps in the conductance as a result of atomic rearrangements, and randomly change the resulting electron interference. The continuous change of $\partial G / \partial V$ during elastic deformation of the contact along a plateau results from the gradual elongation of the electron path lengths, and hence in a gradual change of the resulting interference. In Fig. 3 the open squares represent the points at steps in the conductance and $\partial G / \partial V$ which have been excluded from the statistical analysis by the selection procedure discussed above. As can be seen in the figure, the excluded data consist exclusively of the last and first points on a plateau, together with points which lie between two plateaus as a result of the finite integration time of the lock-in amplifiers.

Figure 4 shows the distribution of values measured for $\partial G / \partial V$ in a particular range of conductance collected from 3500 individual curves similar to the one presented in Fig. 3. The distribution at $1 G_{0}$ is clearly much narrower than the other two at noninteger values. This is statistical evidence for what was already observable in measurements of the bias dependence of the conductance for a single contact in Fig. 2(b). We will argue that the narrow distribution can be ex- 


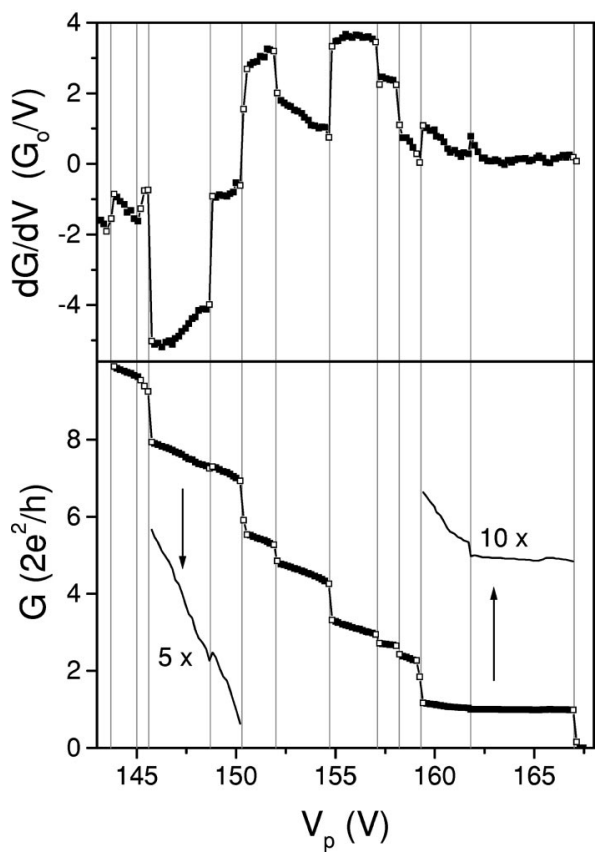

FIG. 3. Typical example of the simultaneous measurement of voltage dependence of the conductance $\partial G / \partial V$ and the conductance $G$, as a function of piezovoltage $V_{P}$ for gold measured with a constant modulation amplitude of $20 \mathrm{mV}$. The graph includes vertical gray lines which show that the steps in both quantities coincide. Two plateaus have been enlarged and offset to show the tiny steps in the conductance. The open squares represent the points excluded from the ensemble average by the selection procedure. The elongation of the contact is linear with $V_{P}$, and $10 \mathrm{~V}$ corresponds to about $1 \mathrm{~nm}$.

plained by a suppression of the conductance fluctuations [Eq. (8)], as a result of $T_{1}$ being approximately equal to 1 and all other $T_{n} \approx 0$. In the presentation of the data below we concentrate on the width of these types of distributions, $\sigma_{G V}$, determined for a fixed number of data points.

The shape of the distribution curve shown in Fig. 4(a) is

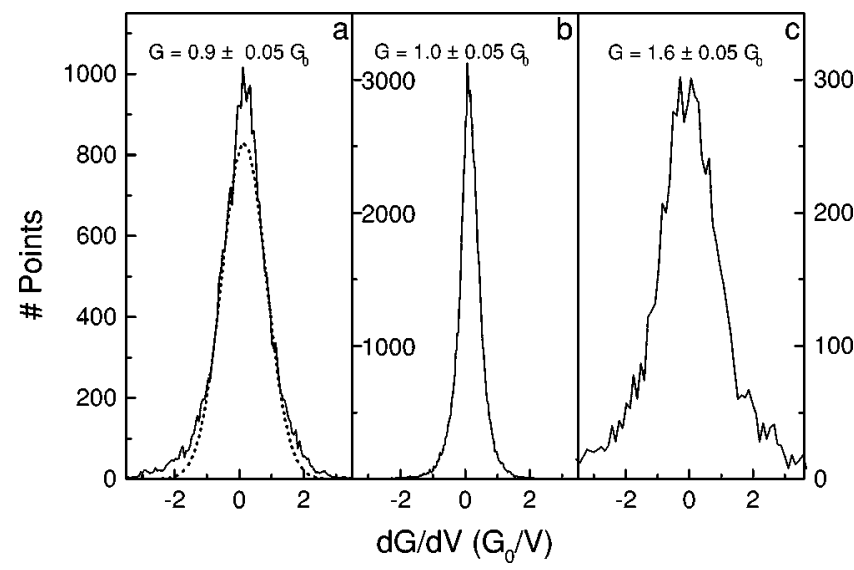

FIG. 4. The distribution of $\partial G / \partial V$ values in a particular conductance range collected from 3500 individual curves for gold such as the one presented in Fig. 3. The conductance range of the three curves roughly corresponds to the conductance of the $\partial I / \partial V$ curves in Fig. 2. (a) $G=0.9 \pm 0.05 G_{0}$. (b) $G=1.0 \pm 0.05 G_{0}$. (c) 1.6 $\pm 0.05 G_{0}$. The dotted curve in (a) represents a Gaussian fit of the data.
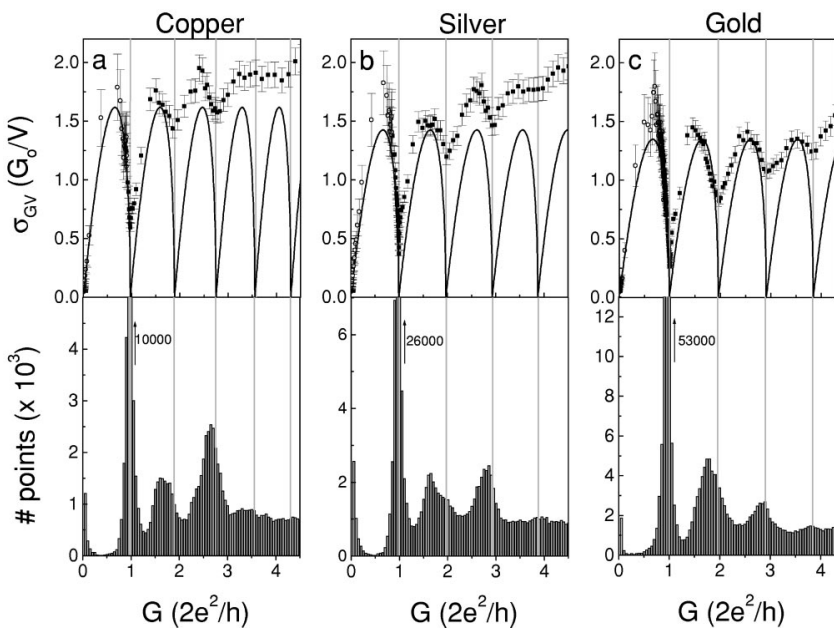

FIG. 5. $\sigma_{G V}$ (top) and conductance histogram (bottom) against $G$ for (a) copper 3000 curves, (b) silver 2400 curves, and (c) gold 3500 curves. The averages have been obtained from 300 data points for each of the open circles and 2000, 2000, and 2500 data points for each of the solid squares for copper, silver, and gold, respectively. The solid curve in each case show the behavior of a single channel opening at a time. The vertical gray lines represent the corrected integer conductance values.

much sharper around the $\partial G / \partial V=0$ value than a Gaussian distribution. The tails of these curves also deviate from Gaussian behavior. These deviating features are analogous to the peaks calculated and measured in the distribution of parametric derivatives (e.g., thermopower) of quantum dots with single-mode ballistic point contacts. ${ }^{27}$ The origin of this cusp at zero amplitude is the limitation of the range over which the differentiated parameter can vary in value. At both of its maximal values the parametric derivative is zero, leading to an enhancement of the statistics at zero amplitude.

In the upper panels of Fig. 5, we present the measured $\sigma_{G V}$ for the noble metals copper, silver, and gold. The data points for $\sim 3000$ individual curves such as the one in Fig. 3 were sorted as a function of conductance. From this total collection of data points the root-mean-square of $\partial G / \partial V$ was calculated for groups of 300,2000 , or 2500 successive data points, depending on the density of points available. This total collection of data points was also used to calculate the corresponding conductance histograms plotted in the lower panels of the figure.

The electronic properties of these three noble metals are very similar, which is reflected in the similar behavior we obtain for $\sigma_{G V}$ as a function of conductance. Minima in $\sigma_{G V}$ near $1 G_{0}, 2 G_{0}$, and $3 G_{0}$ can be observed in all three cases. The minima, however, are most pronounced for gold which even has a small dip near $4 G_{0}$. Another important similarity, as is apparent from the peaks in the conductance histogram, is the preferred values for the conductance just below $1 G_{0}$, $2 G_{0}$, and $3 G_{0}$ for all three materials. ${ }^{15-19}$

When comparing the experimental results with our model it is important to note that a given value for $G=G_{0} \Sigma_{n=1}^{N} T_{n}$ can be constructed in many ways from a choice of transmission values $\left\{T_{n}\right\}$. The experimental values for $\sigma_{G V}$ are, therefore, an average over impurity configurations and transmission values. Assuming the averages are independent, we can compare the data with various choices for the distribu- 
tion of the transmissions. In each upper panel of Fig. 5 the solid curve depicts the behavior of Eq. (8) for a single channel opening at a time, i.e., in the interval $G / G_{0} \in\{0,1\}$ there is a single channel contributing to the conductance with $G$ $=G_{0} T_{1}$, in the interval $\{1,2\}$ there are two channels with one fully open $G=G_{0}\left(1+T_{2}\right)$, etc. The curves in the figure have been scaled so they best fit the data. From this scaling an estimate for the mean free path can be obtained when a reasonable value range ${ }^{28}$ for the opening angle $\gamma=35^{\circ}-50^{\circ}$ is assumed. For copper, silver and gold, we obtain a value of $l_{e}=3 \pm 1 \mathrm{~nm}, l_{e}=4 \pm 1 \mathrm{~nm}$, and $l_{e}=4 \pm 1 \mathrm{~nm}$, respectively.

For gold the description of the experimental data with a single-channel opening at a time works surprisingly well. In particular, for the minimum near $1 G_{0}$, and for the fact that the maximal values between the integer conductance values are all nearly equal. The main discrepancy is that the minima become less pronounced for higher conductances. The welldeveloped structure observed in $\sigma_{G V}$, with a dependence which closely follows the $\sqrt{\Sigma T_{n}^{2}\left(1-T_{n}\right)}$ behavior of Eq. (8), demonstrates a property of the contacts which we refer to as the saturation of the channel transmission ${ }^{9}$ : there is a strong tendency for the channels contributing to the conductance of atomic-size contacts of gold to be fully transmitting, with the exception of one, which then carries the remaining fractional conductance.

For copper and silver the amplitude of the data increases together with the degradation of the minima. These two metals also exhibit the saturation of the channel transmission effect, but clearly not as rigorous as for gold. This reflects itself in the estimates we can make for the contribution of an additional channel at the first three minima. Neglecting the small contribution of the higher-order terms in $a_{l, r_{m n}}$, these are $2 \%, 12 \%$, and $15 \%$ for copper, $1 \%, 11 \%$, and $18 \%$, for silver, and $0.5 \%, 6 \%$, and $10 \%$ for gold. The concept of the saturation of transmission channels is consistent with the model of Cuevas et al. ${ }^{7}$ and other recent experimental work, which shows that, for gold, the conductance at $G=1 G_{0}$ of a single atom is carried by a single mode. ${ }^{8,11}$

The minima in $\sigma_{G V}$ lie at values for $G$ below the integer conductance values. This shift is due to the scattering of transmitted electrons back to the contact, which apart from a fluctuating first-order contribution in $a_{l, r_{m n}}$ which determines $\partial G / \partial V$, also gives rise to a shift in $\langle G\rangle$ when contributions to second order in $a_{l, r_{m n}}$ are taken into account. Ideally we would like to plot $\sigma_{G V}$ as a function of $\Sigma_{n=1}^{N} T_{n}$, with $T_{n}$ the transmission probability of mode $n$ of the bare contact, but the bare contact is always measured in series with the diffusive banks. In order to correct for the backscattering to lowest order, the theoretical curves have been plotted as a function of $G=G_{0} \Sigma_{n=1}^{N} T_{n}\left[1-\sum_{m=1}^{N} T_{m}\left(\left\langle\left|a_{l_{m n}}\right|^{2}\right\rangle+\left\langle\left|a_{r_{m n}}\right|^{2}\right\rangle\right)\right]$, where $\left\langle\left|a_{l, r_{m n}}\right|^{2}\right\rangle$ have been adjusted for optimal agreement with the experimental minima. The vertical gray lines in each figure represent the corrected integer conductance values using this lowest-order procedure. For gold and silver this value is comparable: $\left\langle\mid a_{l, r_{m n}}{ }^{2}\right\rangle=0.005$ versus 0.004 , respectively, and hence is consistent with the similar amplitude of $\sigma_{G V}$ observed for both materials. Copper has a somewhat larger amplitude for $\sigma_{G V}$, in accordance with a larger shift in the conductance minima with $\left\langle\left|a_{l, r_{m n}}\right|^{2}\right\rangle=0.014$. We can relate this value for the conductance correction to a rough estimate for the mean free path by equating $\left\langle\left|a_{l, r_{m n}}\right|^{2}\right\rangle$ to the integral of Eq. (6) over all path lengths. As a lower integration limit we have taken the typical shortest path time $\tau_{e}$, for the upper limit infinity, and for the opening angle we have assumed the typical range $35^{\circ}-50^{\circ}$. The value for $l_{e}$ we obtain using this method is $4 \pm 1,7 \pm 1$, and $6 \pm 1 \mathrm{~nm}$ for copper, silver, and gold, respectively. This is quite close to the mean free path derived above from the amplitude of $\sigma_{G V}$, and is therefore in accordance with our model. The values for the mean free path we obtain are much shorter than what is normally found for bulk samples, and can probably be attributed to surface scattering near the contact. Assuming surface scattering is indeed responsible, an important property of the mean free path which we neglect here is that $l_{e}$ will not be a constant as a function of the conductance, but rather increase as the contact diameter becomes larger. This size dependence of the mean free path is not expected to be very significant in the range of validity of our model, where we assume a contact diameter $d<L<l_{e}$.

The relatively short $l_{e}$ we obtain is responsible for the correction to the quantized conductances, but it is too long to hold backscattering responsible for the measurement of the significant frequency with which nonquantized values are measured. Also, if scattering is held primarily responsible for reducing the conductance from for instance a perfect conductance of $2 G_{0}$ to $1.5 G_{0}$, then it is not unreasonable to assume that contacts with a perfect conductance of $1 G_{0}$ are reduced to $0.5 G_{0}$ with a probability of the same order of magnitude. This is not observed experimentally at low temperatures, as contacts with a conductance of $0.5 G_{0}$ occur more than 500 times less frequently for silver and copper than contacts with a conductance of $1.5 G_{0}$ (the formation of atomic chains ${ }^{29}$ reduces this ratio to about 20 times in the case of gold, since the conductance of the chains is quite sensitive to distortions making contacts with a conductance of $0.5 G_{0}$ occur with an enhanced frequency). If, on the other hand, one assumes that contribution from tunneling, due to for instance geometrical considerations, are more important, the appearance of nonquantized values above $1 G_{0}$ finds a natural explanation. The formation of geometries with a conductance smaller than $1 G_{0}$ is highly unlikely since the smallest contact geometry is that of a single atom with conductance $1 G_{0}$, and, when the contact breaks, the banks relax back preventing high transmission probability tunneling contributions from contributing. The latter process is usually referred to as the jump to tunneling. ${ }^{3}$

An important feature for all three noble metals is that the minima in $\sigma_{G V}$ near $2 G_{0}$ do not coincide with the respective peaks in the histograms. The minima lie at the expected conductances based on the backscattering amplitude we require to consistently fit all the minima, the so-called corrected integer conductance values. The second peak in the histograms clearly are located at lower conductance values. We propose that this discrepancy is caused by favorable atomic configurations which have a bare conductance smaller than $2 G_{0}$, and thus give rise to a peak in the histogram below the corrected conductance value for $2 G_{0}$. 


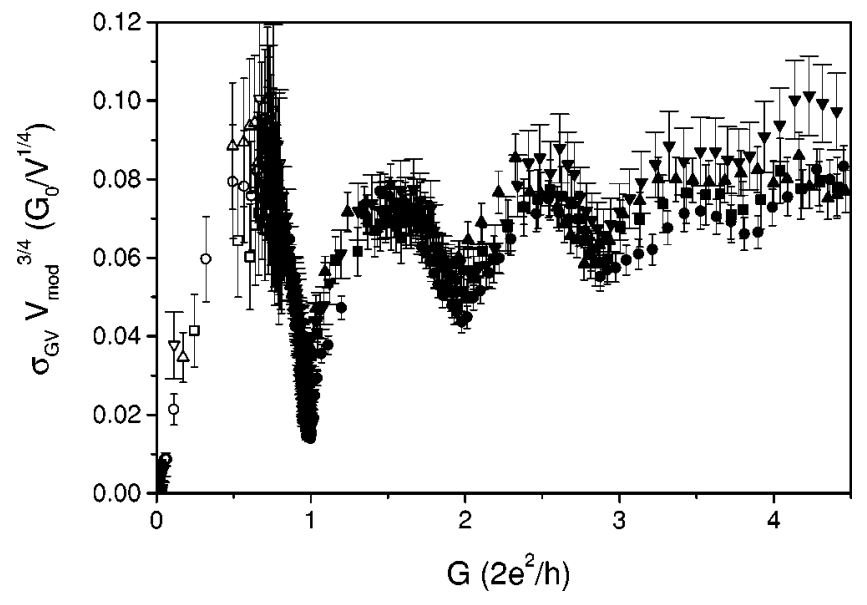

FIG. 6. $\sigma_{G V}$ multiplied by the modulation amplitude to the power 3/4 against conductance $G$ for gold measured at (squares) 10 $\mathrm{mV}$, (circles) $20 \mathrm{mV}$, (up triangles) $40 \mathrm{mV}$, and (down triangles) 80 $\mathrm{mV}$.

\section{TEST OF THE MODEL}

Apart from the set of transmission values $\left\{T_{n}\right\}$ the equation contains two free parameters $l_{e}$ and $\gamma$ over which we do not have any experimental control. The dependence on the modulation voltage $V_{\text {mod }}$, however, is a parameter which we can verify, assuming that all other relevant parameters are independent of the applied voltage. This is a reasonable assumption for the modulation amplitudes at which we measure, as parameters like $\left\{T_{n}\right\}$ vary on the scale of $E_{F}$, and $l_{e}$ and $\gamma$ are not expected to change on the scale of $100 \mathrm{mV}$. Assuming further that $e V_{\text {mod }}>\hbar / \tau_{\phi}$ then the product $\sigma_{G V} V_{m o d}^{3 / 4}$ should be constant for all $V_{\text {mod }}$.

In Fig. 6, $\sigma_{G V} V_{m o d}^{3 / 4}$ has been plotted against $G$, for $V_{\text {mod }}$ $=10,20,40$, and $80 \mathrm{mV}$. Within the experimental accuracy no modulation voltage dependence is observed, as all four data sets coincide very well. This would not be the case unless the power of the modulation amplitude dependence is close to $3 / 4$. Using a procedure that calculates the minimal difference between the six combinations of experimental curves, which have been multiplied by their respective modulation amplitudes to a power which is the free parameter, we find this power to be $0.71 \pm 0.06$, in good agreement with the $3 / 4$ predicted by the theory.

The mechanism used to describe the fluctuations in the conductance above also produces fluctuations in other transport properties, notably the thermopower. Measuring the thermopower of atomic-size metallic contacts requires a completely different experimental method, and is performed on an energy scale much smaller than that necessary for determining $\sigma_{G V}$. The experimental results, ${ }^{30}$ however, have been successfully described by a theory based on the same principles as those presented above. The predicted theoretical relationship between the standard deviation of the thermopower $\sigma_{S}$ and $\sigma_{G V}$ is given by

$$
\sigma_{G V}=\sigma_{S} \frac{2.71 e^{2} G_{0} \sum_{n=1}^{N} T_{n}}{c k_{B}\left(k_{B} \theta\right)^{1 / 4}\left(e V_{m o d}\right)^{3 / 4}},
$$

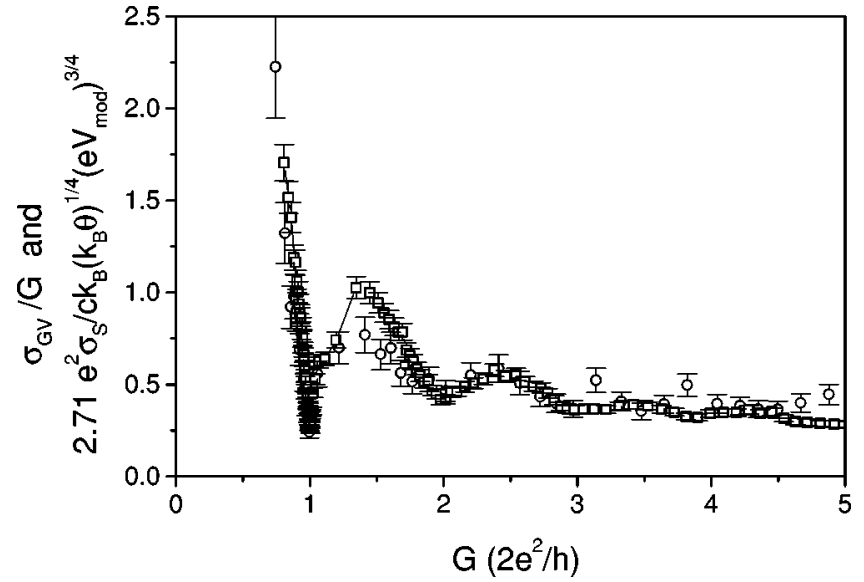

FIG. 7. Comparison of the standard deviation of the thermopower $\sigma_{S}$ and the standard deviation of the voltage dependence of the conductance $\sigma_{G V}$ by plotting $(\bigcirc)$ $2.71 e^{2} \sigma_{S} / c k_{B}\left(k_{B} \theta\right)^{1 / 4}\left(e V_{m o d}\right)^{3 / 4}$ and $(\square) \sigma_{G V} / G$ as a function of conductance.

where the numerical constant $c=5.94$. The only parameters in the above relation are $\theta$, the temperature at which the thermopower measurements were performed; $V_{\text {mod }}$, the modulation amplitude for the conductance fluctuation measurements; and the total conductance $G_{0} \sum_{n=1}^{N} T_{n}$. All unknown parameters in Eq. (8), the set of transmission probabilities $\left\{T_{n}\right\}, l_{e}$ and $\gamma$ cancel out, and the scaling relation provides an independent test of the experimental data.

By comparing $\sigma_{G V}$ to measurements of the thermopower on atomic size contacts ${ }^{30}$ using Eq. (11), we can effectively extend the energy range over which we test our model to an order of magnitude smaller, and test the experimental procedure against a completely independent method. In Fig. 7 we have plotted $2.71 e^{2} \sigma_{S} / c k_{B}\left(k_{B} \theta\right)^{1 / 4}\left(e V_{m o d}\right)^{3 / 4}$ and $\sigma_{G V} / G$ as a function of conductance. We have set $\theta=12 \mathrm{~K}$ and $V_{\text {mod }}=20 \mathrm{mV}$ in accordance with the experimental conditions. Excellent agreement is obtained between both experimental methods without any free parameters. We interpret this as a successful test for the validity of the principle on which our theoretical analysis is based. The dependence on the opening angle $\gamma$, however, we regretfully cannot verify experimentally, as we have no control over the contact geometry.

\section{ALKALI METAL SODIUM}

Sodium also is a monovalent metal, but its histogram determined from 1800 curves (lower panel, Fig. 8), is completely different from that observed for copper, silver, or gold. The statistically preferred conductance values are observed as peaks in the histogram ${ }^{15}$ near $1 G_{0}, 3 G_{0}, 5 G_{0}$, and $6 G_{0}$ rather than near $1 G_{0}, 2 G_{0}$, and $3 G_{0}$. This series of peaks in the histogram at $1 G_{0}, 3 G_{0}, 5 G_{0}$, and $6 G_{0}$ have been interpreted as resulting from the quantization of the conductance in a cylindrically shaped nanowire. The histogram peaks are very sharp, and in the 1800 curves measured almost no data is obtained between $0 G_{0}$ and $1 G_{0}$ and between $1 G_{0}$ and $2 G_{0}$. For this reason no points for $\sigma_{G V}$, determined from the same 1800 curves, are presented in these ranges (upper panel Fig. 8). Even with these points 


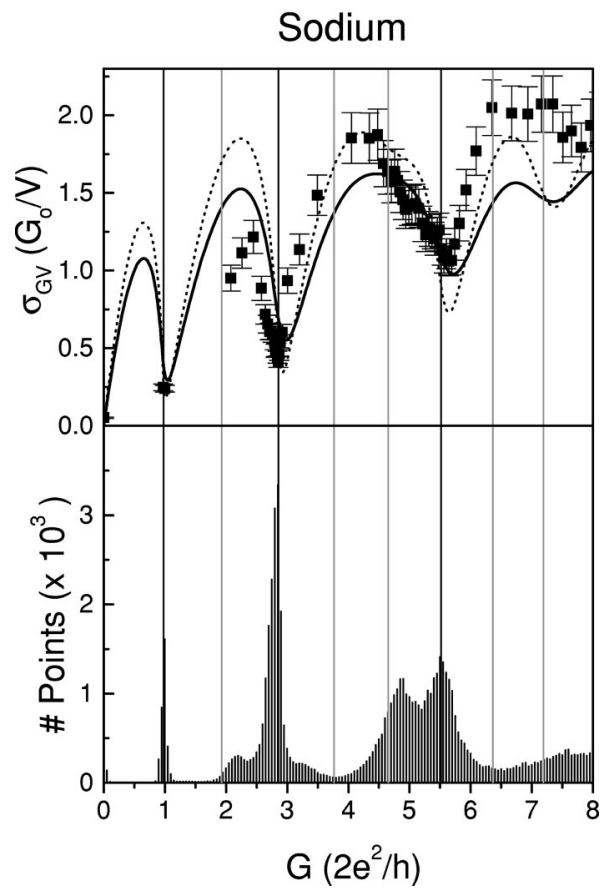

FIG. 8. $\sigma_{G V}$ (top) and conductance histogram (bottom) against $G$ for 1800 sodium curves with each solid square representing the statistics on 1000 data points. The vertical black and gray lines indicate the corrected integer conductance values for which the histogram peaks, respectively, do and do not correspond with minima in $\sigma_{G V}$. The curves depict the behavior of a hyperbolic constriction in a three-dimensional electron gas with circular aperture, with (-) opening angle $\gamma=60^{\circ}$ and mean free path $l_{e}=4.4 \mathrm{~nm},(\cdots) \gamma$ $=45^{\circ}$ and $l_{e}=5 \mathrm{~nm}$.

absent, the $\sigma_{G V}$ measured for sodium is distinctly different from that observed for the noble metals. In $\sigma_{G V}$ we observe definite minima near $3 G_{0}$ and $6 G_{0}$ and although there has been no data measured in the ranges $0<G<1 G_{0}$ and 1 $<G<2 G_{0}$, the value of $\sigma_{G V}$ at $1 G_{0}$ is small making it a very probable location of a minimum. Since there are no data below $2 G_{0}$, we cannot exclude that there is a small minimum at $2 G_{0}$.

The histogram peaks coincide with the minima in $\sigma_{G V}$, with the exception of the peak near $5 G_{0}$. The absence of a minimum at $5 G_{0}$ is at first surprising. When one considers that in a conductance histogram for a model of a threedimensional cylindrical contact based purely on a freeelectron gas, the peak at this value is found to be nearly absent due to smearing by tunneling contributions, ${ }^{31}$ it is striking that a histogram peak is there at all. Also, unlike the other peaks, the one below $5 G_{0}$ and also the small one above $2 G_{0}$ do not coincide with the corrected integer conductance values. We propose that these two peaks result from favorable atomic configurations, which are sampled more often than other conductance values while making a histogram, but do not result from stable quantized conductance values determined by an integer number of nearly open channels.

As in the case of the noble metals there is a systematic shift of the position of the minima in $\sigma_{G V}$ to lower conductance values. The corrected integer conductance multiples are shown in Fig. 8 as vertical gray and black lines depending on whether they coincide with the minima in $\sigma_{G V}$ or not. For the correction to the integer conductance values in Fig. 8 we have used Eq. (9) with $r=0.015$. This value corresponds to a series resistance of $200 \Omega$ for $g \rightarrow \infty$ and $400 \Omega$ for $g$ $=1$ as is evident from Eq. (10). Equation (9) is used, rather than the first-order correction applied to the noble metals, because the conductance extends to larger values where higher-order terms in the backscattering amplitude become relevant.

We want to compare these results with the simplest possible model, which neglects the atomic character of the contact and only takes the cylindrical symmetry and finite length of the contact into account. For this purpose we have made use of the calculations by Torres et al. ${ }^{32}$ The model consists of a free-electron gas confined by hard-wall boundaries, which have the form of a hyperboloid. The differential equations for this system can be solved numerically, from which the transmission probabilities $T_{n}$ for each mode as a function of the contact diameter can be obtained. This makes a direct evaluation of Eq. (8) possible, where the only remaining adjustable parameter is the mean free path. The opening angle which describes the shape of the hyperboloid, and thus the dependence of the mode transmissions on the contact diameter, is the same as the one which enters into Eq. (8). We have added two $\sigma_{G V}$ curves calculated for such a system to the graph. For curves in the opening angle range from $60^{\circ}$, with mean free path $l_{e}=4.4 \mathrm{~nm}$, to $45^{\circ}$, with $l_{e}=5 \mathrm{~nm}$, we find reasonable agreement between various ranges in the data and the theoretical curve.

The differences between the calculated curve and the measured data can be attributed to the averaging over many contact geometries and thus over a range of $\gamma$ values. Also, the smearing in the conductance $\langle G\rangle$ due to the ensemble average of defect configurations is not included. This property will make the minima less deep and sharp but will hardly influence the maxima.

Another feature of the calculated curve, which can also be recognized in the measured data, is that the minimum in the experimental and calculated $\sigma_{G V}$ below $6 G_{0}$ does not coincide exactly with the corrected quantized value for the conductance, even after application of the same series resistance we have used to compensate for the shift in the histogram peaks. In other words, when we ignore the series resistance correction, the model predicts the minima to be shifted above the integer values. This is a direct result of the significant tunneling contributions for the opening angles we have used to model the contact when just opening a mode in combination with the asymmetry of the dependence of $\sigma_{G V}$ on the mode transmission. This systematic shift becomes more pronounced with larger opening angles, larger conductance values, and the presence of degenerate modes. The value of the opening angle we obtain for sodium from the theoretical curves, $\gamma=45^{\circ}-60^{\circ}$ is comparable to but somewhat larger than the typical estimates made for the opening angles of atomic size gold contacts. ${ }^{28}$

\section{TRIVALENT METAL ALUMINUM}

The statistically preferred conductance values for aluminum are shown in the lower panel of Fig. 9. The clear peaks, evident in the histogram below $1 G_{0}$ and $2 G_{0}$ and a weak bump above $3 G_{0}$ are in accordance with previous measurements. ${ }^{20}$ The peaks are less pronounced, but at first 


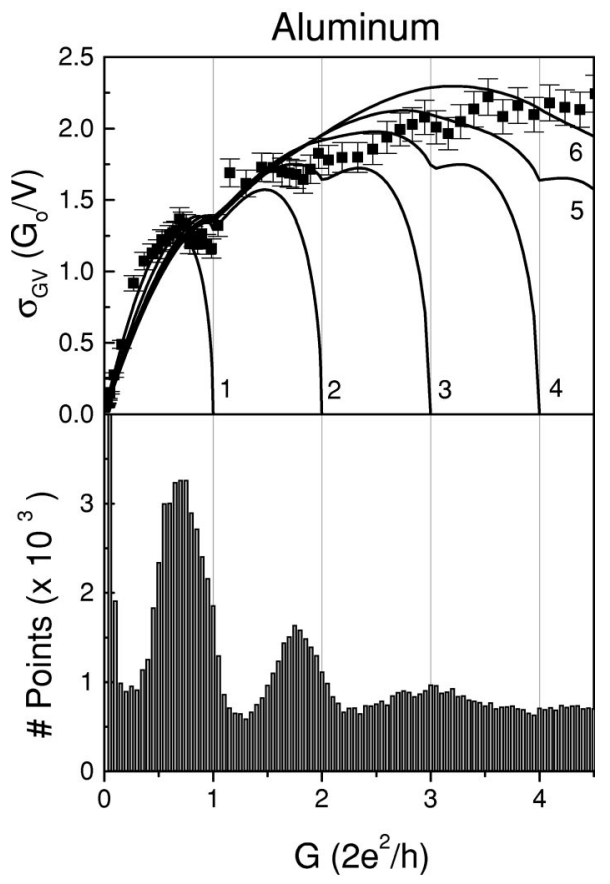

FIG. 9. $\sigma_{G V}$ (top) and conductance histogram (bottom) against $G$ for 2800 aluminum curves with each solid square representing the statistics on 2000 data points. The curves in the graph have been labeled with a number and represent the contribution to $\sigma_{G V}$ of (1) a single channel opening, a random distribution over (2) two, (3) three, (4) four, (5) five, and (6) six channels (see text).

glance similar to those observed for gold, silver, and copper. The most important discrepancy between the monovalent metals and aluminum is that in the latter case the first peak is broader and clearly displaced below $1 G_{0}$.

The measured $\sigma_{G V}$ for aluminum, presented in the upper panel of Fig. 9, is completely different from the behavior observed for the noble metals copper, silver, and gold. The clear minima at $1 G_{0}, 2 G_{0}$, and $3 G_{0}$ have been replaced by a slight dip at $1 G_{0}$. In order to understand these measured features it is important to realize that a single aluminum atom has a conductance close to $G_{0}$ but admits three conductance channels. ${ }^{8,10}$ It is thus not surprising that the behavior associated with the saturation of a single partially open channel is not observed for aluminum. The histogram peaks observed for this trivalent material can thus be attributed to another mechanism. A likely candidate is favorable atomic configurations, which are probed more frequently than others.

In Fig. 9 a series of curves are included which show the behavior for a single channel, and a random distribution of two, three, four, five, and six channels. The curves have been generated by calculating the square root of $\sigma_{\max }^{2} \int_{0}^{1} \cdots \int_{0}^{1} P\left(T_{1}, T_{2}, \ldots, T_{N}\right) \sum_{n=1}^{N} T_{n}^{2}\left(1-T_{n}\right) d T_{1} \cdots d T_{N}$ where $P$ is the probability distribution giving an equal probability to every transmission value for each $T_{n}$ under the constraint that $\sum_{n=1}^{N} T_{n}=G / G_{0}$. These curves have been labeled $1,2,3,4,5$, and 6 , respectively, and have all been scaled with the same amplitude. The dips in the calculated curves with a random distribution of two or more channels results from the property that, in a random distribution, at multiples of the conductance quantum, there is a finite probability to encounter some $T_{n}=(0$ or 1$)$, for which their contribution to $\sigma_{G V}=0$. This effect, however, and hence the dip, becomes less pronounced with an increasing number of channels. For the lowest conductances $\left(<0.5 G_{0}\right)$ one can observe that the behavior most closely follows that of a single channel. This is expected, as for small conductances in the tunneling regime a single channel is expected to dominate the conductance and, hence, the behavior in $\sigma_{G V}$. As the conductance increases the behavior becomes more like a random distribution over an increasing number of channels. The random distribution of channels which we have introduced serves only to illustrate that the small dip at $1 G_{0}$ appears for a limited number of channels even without the saturation of channel transmission effect, and that the gradual increase in $\sigma_{G V}$ is a direct result of more channels contributing for larger conductances. The actual behavior of the transmission channels is probably not completely random as can be judged from the theory, ${ }^{7}$ which shows that there is usually one dominant channel and two smaller ones for a single aluminum atom. Nonetheless the curves reproduce the evolution of $\sigma_{G V}$ as a function of conductance with reasonable accuracy.

\section{TRANSITION METALS NIOBIUM AND IRON}

The measured $\sigma_{G V}$ (upper panel) and histogram (lower panel) for 2400 niobium curves, recorded at a temperature of $10 \mathrm{~K}$ in order to avoid effects of the superconductivity on the voltage dependence of the conductance, have been plotted in Fig. 10(a). For iron the measurements of $\sigma_{G V}$ (upper panel) and the histogram (lower panel) recorded for 700 curves are presented in Fig. 10(b).

Both $s d$ metals, niobium and iron, show completely different features when compared to the other materials we have discussed so far. When compared with each other, the measurements for niobium and iron are so similar that they nearly are indistinguishable. For both metals, $\sigma_{G V}$ increases strongly from $0 G_{0}$ to $1 G_{0}$ and above this conductance value, increases only slightly. The dip observed in $\sigma_{G V}$ for aluminum is absent and the increase in $\sigma_{G V}$ with conductance is much smaller than was the case with aluminum. From the single peak in the histogram we can deduce that both materials have a statistically preferred conductance value just above $2 G_{0}$. This peak is expected to be the result of the reproducibility in the conductance of the last plateau consisting of a single niobium or iron atom. In the case of niobium this value is in excellent agreement with the measured and calculated conductance value for a single atom of niobium $\left[(2-3) G_{0}\right] .^{7,8}$ For iron these calculations and measurements have not yet been performed.

The completely random distribution used to describe the behavior of aluminum clearly cannot reproduce the measured $\sigma_{G V}$ for niobium and iron. If one considers distributions for the transmission probabilities that are closer to the five calculated transmission probabilities contributing to a singleatom contact, ${ }^{7}$ the experimental behavior up to $3 G_{0}$ can in principle be simulated. The number of free parameters in such an analysis, however, makes such an ad hoc procedure quite meaningless. More theoretical work must be performed to provide an approximation for the range of transmission channel distributions that should be considered in order for the measurements to be reliably related to a general trend. 

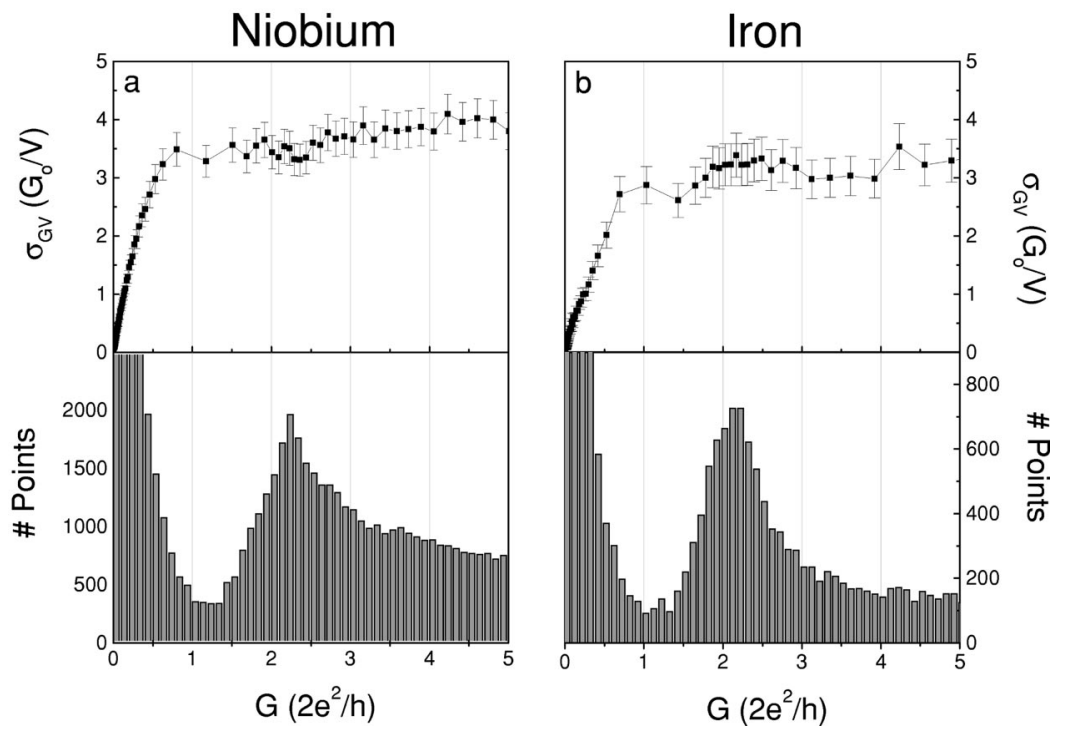

FIG. 10. (a) $\sigma_{G V}$ (top) and conductance histogram (bottom) against $G$ for 2400 niobium curves measured at $10 \mathrm{~K}$ with each solid square representing the statistics on 1000 data points. (b) $\sigma_{G V}$ (top) and conductance histogram (bottom) against $G$ for 700 iron curves with each solid square representing the statistics on 500 data points.
The fact that the measured $\sigma_{G V}$ above $1 G_{0}$ is almost not dependent on the total conductance suggests that the number of partially open channels contributing to the conductance does not depend on the contact size.

The histogram we have recorded for iron is different from the histogram constructed from 80 iron contacts recorded under ambient conditions in the magnetically saturated state. ${ }^{33}$ Possibly the different temperature at which the experiments are performed or the magnetization state are responsible for the discrepancy. The influence of these experimental conditions should be studied in more detail in the future.

\section{CONCLUSIONS}

With the technique of ensemble-averaged conductance fluctuations, we are able to measure the average properties of the conductance mode evolution of atomic-size contacts. We have successfully tested the modulation voltage dependence of the theory, and can relate $\sigma_{G V}$ to the standard deviation in the thermopower without any free parameters. An important property of the measurement of the conductance fluctuations is that it is not dependent on preferential contact configurations.

Sodium, the most free-electron-like material studied, exhibits electronic behavior which can be reproduced reasonably well with a hyperbolic constriction in a threedimensional electron gas with a circular orifice. The conductance histogram of this material, however, contains contributions from such a circular constriction in a threedimensional electron gas as well as other peaks possibly resulting from favorable atomic configurations. The conductance properties of the other monovalent materials such as gold, silver, and copper seem to be best described by the tendency of the conductance channels to open one by one, a property which has been called the saturation of channel transmission. ${ }^{9}$ The conductance histogram of these materials contains features which seem to coincide with the evolution of the conductance modes, but particularly the second peaks in the histograms are also determined by other statistical (probably atomic) properties of the contact. For aluminum, niobium, and iron we find the behavior for $\sigma_{G V}$ we expect based on the atomic-orbital model. ${ }^{7,8,10}$ The conductance histograms of these three materials seem to be dominated by the statistical distribution of atomic configurations.

The concept of saturation of channel transmission was been introduced in Ref. 9, to make a marked distinction between the properties of the conductance modes we observe here, and the statistically preferred conductance values which in the literature are generally referred to as conductance quantization. Indeed, both are based on the quantummechanical Landauer-Büttiker formalism, but the latter assumes there is a statistical preference for contacts with quantized values as a result of this formalism. Since it is not clear to what extent favorable atomic configurations are responsible for the histogram peaks, we feel a sharp distinction should be made between results that can be influenced by favorable atomic positions possibly mimicking the features of conductance quantization, and results which truly probe the electronic properties of the contact. With the saturation of the channel transmission we wish only to describe the evolution of these modes, but we do not rule out that conductance quantization may prove to be an important factor which influences the contact formation. ${ }^{34}$ However, in view of the results presented in Figs. 9 and 10 together with the arguments presented above for the other materials, we should be aware that peaks in a histogram by themselves give no unambiguous information about the actual composition of the conductance modes.

For the correction to the bare contact conductance due to scattering near the contact, we find that this correction is correlated to the amplitude of the conductance fluctuations and hence the elastic mean free path. This provides strong experimental evidence that these types of scattering effects are indeed responsible for the so-called series resistance.

\section{ACKNOWLEDGMENTS}

This work is part of the research program of the "Stichting FOM,' which is financially supported by NWO. The development of the theory and interpretation of the gold data was done in collaboration with C. Urbina, D. Esteve and M. Devoret. We acknowledge the stimulating support of L.J. de Jongh. 


\section{APPENDIX}

Incorporating a finite modulation voltage into the expression for $\sigma_{G V}$ is somewhat more technical. In analogy with Eq. (5), we can write, for the energy-dependent part of the current,

$$
\delta I=\frac{2 e}{h} \int_{0}^{e V} \sum_{n=1}^{N} T_{n} 2 \operatorname{Re}\left[r_{n} a_{l_{n n}}(-E)+r_{n}^{\prime} a_{r_{n n}}(e V-E)\right] d E .
$$

We must include the modulation voltage $V=V_{0}$ $+V_{\text {mod }} \sin \left(\omega_{0} \tau^{\prime}\right)$ in the argument for the backscattering amplitude, which we write as an integral over contributions of path traversal times $\tau$. Hence $a_{l, r_{n n}}(E)$ $=\int a_{l, r_{n n}}(\tau) \exp [-i E \tau / \hbar] d \tau$, where we assume the dominant energy dependence is the phase factor. We will first consider the contribution to the current of a single path with time $\tau$ : $\delta I(\tau)$. At a later stage we will perform the integration over different paths. Evaluating the integral over $E$ in Eq. (A1) gives

$$
\begin{aligned}
\delta I(\tau)= & \text { const. }+\frac{2 e}{h} 2 \sum_{n=1}^{N} T_{n} \frac{\hbar}{\tau} \\
& \times \operatorname{Re}\left[-i r_{n} a_{l_{n n}}(\tau) e^{i e V_{0} \tau / \hbar} e^{+i e V_{m o d} \tau \sin \left(\omega_{0} \tau^{\prime}\right) / \hbar}\right. \\
& \left.+i r_{n}^{\prime} a_{r_{n n}}(\tau) e^{-i e V_{0} \tau / \hbar} e^{-i e V_{m o d} \tau \sin \left(\omega_{0} \tau^{\prime}\right) / \hbar}\right] .
\end{aligned}
$$

We are measuring the ac component of the current at twice the modulation frequency, this is equivalent to measuring the second derivative of the current with respect to voltage. Therefore we expand the exponential term Eq. (A2) in harmonics of the modulation frequency,

$$
\begin{aligned}
e^{i e V_{\text {mod }} \tau \sin \left(\omega_{0} \tau^{\prime}\right) / \hbar} & \\
= & \sum_{n=-\infty}^{\infty} e^{i n \omega_{0} \tau^{\prime}} J_{n}\left(e V_{m o d} \tau / \hbar\right) \\
= & J_{0}\left(e V_{m o d} \tau / \hbar\right)+2 i \sin \left(\omega_{0} \tau^{\prime}\right) J_{1}\left(e V_{m o d} \tau / \hbar\right) \\
& +2 \cos \left(2 \omega_{0} \tau^{\prime}\right) J_{2}\left(e V_{m o d} \tau / \hbar\right)+\cdots,
\end{aligned}
$$

where $J_{n}(z)$ is the $n$th Bessel function. In the last step we used $J_{-n}(z)=(-1)^{n} J_{n}(z)$. We are particularly interested in the last term in Eq. (A3), which we will use to obtain the part of the current which is proportional to twice the modulation frequency $\left[\cos \left(2 \omega_{0} \tau^{\prime}\right)\right]$,

$$
\begin{aligned}
\delta I_{2 \omega_{0}}(\tau)= & -\frac{2 e}{h} 4 \sum_{n=1}^{N} T_{n} \frac{\hbar}{\tau} \cos \left(2 \omega_{0} \tau^{\prime}\right) J_{2}\left(e V_{m o d} \tau / \hbar\right) \\
& \times \operatorname{Re}\left[-i r_{n} a_{l_{n n}}(\tau) e^{i e V_{0} \tau / \hbar}+i r_{n}^{\prime} a_{r_{n n}}(\tau) e^{-i e V_{0} \tau / \hbar}\right] .
\end{aligned}
$$

In general, the Taylor expansion of the current for small modulation amplitude is

$$
\begin{aligned}
I\left[V_{0}+V_{\text {mod }} \sin (\omega t)\right]= & I\left(V_{0}\right)+\left(\frac{\partial I}{\partial V}\right)_{V_{0}} \sin (\omega t) \\
& -\frac{1}{4}\left(\frac{\partial^{2} I}{\partial V^{2}}\right) V_{m o d}^{2} \cos \left(2 \omega_{0} \tau^{\prime}\right)+\cdots
\end{aligned}
$$

Combining Eqs. (A1), (A4), and (A5) we obtain

$$
\begin{aligned}
\left(\frac{\partial^{2} I}{\partial V^{2}}\right)_{V_{0}}= & -\int_{0}^{\infty} \frac{4}{V_{m o d}^{2}} \frac{2 e}{h} 2 \sum_{n=1}^{N} T_{n} \frac{\hbar}{\tau} J_{2}\left(e V_{m o d} \tau / \hbar\right) \\
& \times\left[-i r_{n} a_{l_{n n}}(\tau) e^{i e V_{0} \tau / \hbar}+i r_{n}^{\prime} a_{r_{n n}}(\tau) e^{-i e V_{0} \tau / \hbar}\right. \\
& +i r_{n}^{*} a_{l_{n n}^{*}}^{*}(\tau) e^{-i e V_{0} \tau / \hbar} \\
& \left.-i r_{n}^{\prime *} a_{r_{n n}}^{*}(\tau) e^{+i e V_{0} \tau / \hbar}\right] d \tau .
\end{aligned}
$$

We now can continue as before and calculate the correlation function. We assume only terms with the form $a_{l_{n n}}(\tau) a_{l_{n n}}^{*}(\tau)$ or $a_{r_{n n}}(\tau) a_{r_{n n}}^{*}(\tau)$ contribute to this function, as all other combinations of reflection coefficients and combinations of different path times will average to zero, and we introduce the brackets which represent averaging over different impurity configurations:

$$
\begin{aligned}
\sigma_{G V}^{2}= & \left\langle\left(\frac{\partial^{2} I}{\partial V^{2}}\right)^{2}\right\rangle \\
= & \frac{16}{V_{\text {mod }}^{4}}\left(\frac{2 e}{h}\right)^{2} 4 \sum_{n=1}^{N} T_{n}^{2}\left(1-T_{n}\right) \int_{0}^{\infty}\left(\frac{\hbar}{\tau}\right)^{2} J_{2}^{2}\left(e V_{m o d} \tau / \hbar\right) \\
& \times\left\langle a_{l_{n n}}(\tau) a_{l_{n n}^{*}}^{*}(\tau)+a_{r_{n n}}(\tau) a_{r_{n n}}^{*}(\tau)\right\rangle d \tau .
\end{aligned}
$$

Both sides of the contact have the same average properties, and we can write for $\left\langle a_{l_{n n}}(\tau) a_{l_{n n}}^{*}(\tau)\right\rangle=\left\langle a_{r_{n n}}(\tau) a_{r_{n n}}^{*}(\tau)\right\rangle$ $=P_{c l}(\tau)$. Filling in the expression for the classical return probability [Eq. (6)] and substituting $x=e V_{\text {mod }} \tau / \hbar$ gives us

$$
\begin{aligned}
\sigma_{G V}^{2}= & \frac{16}{V_{m o d}^{4}}\left(\frac{2 e}{h}\right)^{2} 16 \sum_{n=1}^{N} T_{n}^{2}\left(1-T_{n}\right) \\
& \times \frac{\hbar^{2} \mathrm{v}_{F}}{2 \sqrt{3 \pi} k_{F}^{2} D^{3 / 2}(1-\cos \gamma)}\left(\frac{e V_{m o d}}{\hbar}\right)^{5 / 2} \int_{0}^{\infty} \frac{1}{x^{7 / 2}} J_{2}^{2}(x) d x .
\end{aligned}
$$

Numerical integration of the integral yields the value 0.03385 , and filling in $D=\mathrm{v}_{F}^{2} \tau_{e} / 3$ finally results in Eq. (8). 
${ }^{1}$ M.F. Crommie, C.P. Lutz, and D.M. Eigler, Science 262, 219 (1993); J.K. Gimzewski and R. Möller, Phys. Rev. B 36, 1284 (1987).

${ }^{2}$ C.J. Muller, J.M. van Ruitenbeek, and L.J. de Jongh, Physica C 191, 485 (1992).

${ }^{3}$ J.M. Krans, I.K. Yanson, Th.C.M. Govaert, R. Hesper, and J.M. van Ruitenbeek, Phys. Rev. B 48, 14721 (1993).

${ }^{4}$ R. Landauer, IBM J. Res. Dev. 1, 223 (1957); M. Büttiker, Phys. Rev. Lett. 57, 1761 (1986).

${ }^{5}$ B.J. van Wees, H. van Houten, C.W.J. Beenakker, J.G. Williamson, L.P. Kouwenhoven, D. Van der Marel, and C.T. Foxon, Phys. Rev. Lett. 60, 848 (1988); D.A. Wharam, T.J. Thornton, R. Newbury, M. Pepper, H. Ahmed, J.E.F. Frost, D.G. Hasko, D.C. Peacock, D.A. Ritchie, and G.A.C. Jones, J. Phys. C 73, 2611 (1994).

${ }^{6}$ G. Rubio, N. Agrait, and S. Vieira, Phys. Rev. Lett. 76, 2302 (1996).

${ }^{7}$ J.C. Cuevas, A. Levy Yeyati, and A. Martín-Rodero, Phys. Rev. Lett. 80, 1066 (1998).

${ }^{8}$ E. Scheer, N. Agrait, J.C. Cuevas, A. Levy Yeyati, B. Ludoph, A. Martín-Rodero, G. Rubio, J.M. van Ruitenbeek, and C. Urbina, Nature (London) 394, 154 (1998).

${ }^{9}$ B. Ludoph, M. Devoret, D. Esteve, C. Urbina, and J.M. van Ruitenbeek, Phys. Rev. Lett. 82, 1530 (1999).

${ }^{10}$ E. Scheer, P. Joyez, D. Esteve, C. Urbina, and M.H. Devoret, Phys. Rev. Lett. 78, 3535 (1997).

${ }^{11}$ H.E. van den Brom and J.M. van Ruitenbeek, Phys. Rev. Lett. 82, 1526 (1999).

${ }^{12}$ P.A.M. Holweg, J.A. Kokkedee, J. Caro, A.H. Verbruggen, S. Radelaar, A.G.M. Jansen, and P. Wyder, Phys. Rev. Lett. 67, 2549 (1991); P.A.M. Holweg, J. Caro, A.H. Verbruggen, and S. Radelaar, Phys. Rev. B 48, 2479 (1993); D.C. Ralph, K.S. Ralls, and R.A. Buhrman, Phys. Rev. Lett. 70, 986 (1993).

${ }^{13}$ B.Z. Spivak and A.Yu. Zyuzin, Mesoscopic Phenomena in Solids, edited by B.L. Altshuler, P.A. Lee, and R.A. Webb (NorthHolland, Amsterdam, 1991).

${ }^{14}$ D.L. Maslov, C. Barnes, and G. Kirczenow, Phys. Rev. Lett. 70, 1984 (1993).

${ }^{15}$ J.M. Krans, J.M. van Ruitenbeek, V.V. Fisun, I.K. Yanson, and L.J. de Jongh, Nature (London) 375, 767 (1995).

${ }^{16}$ M. Brandbyge, J. Schiøtz, M.R. Sørensen, P. Stolze, K.W. Jacobsen, J.K. Nørskov, L. Olesen, E. Lægsgaard, I. Stensgaard, and F. Besenbacher, Phys. Rev. B 52, 8499 (1995).

${ }^{17}$ Z. Gai, Y. He, H. Yu, and W.S. Yang, Phys. Rev. B 53, 1042 (1996).
${ }^{18}$ J.L. Costa-Krämer, N. García, and H. Olin, Phys. Rev. B 55, 12910 (1997).

${ }^{19}$ C. Sirvent, J.G. Rodrigo, N. Agrait, and S. Vieira, Physica B 218, 238 (1996).

${ }^{20}$ A.I. Yanson and J.M. van Ruitenbeek, Phys. Rev. Lett. 79, 2157 (1997).

${ }^{21}$ M. Büttiker, IBM J. Res. Dev. 32, 63 (1988).

${ }^{22}$ M. Brandbyge, M.R. Sørensen, and K.W. Jacobsen, Phys. Rev. B 56, 14956 (1997).

${ }^{23}$ In Eq. 2 of Ref. 9, the square root over the $1-\cos \gamma$ has incorrectly been left out. This error only has consequences for the elastic scattering length extracted from the experimental $\sigma_{G V}$. As will become apparent below, this $l_{e}$ is now in much better agreement with the $l_{e}$ obtained from the shift in the integer conductance values.

${ }^{24}$ P. García-Mochales and P.A. Serena, Phys. Rev. Lett. 79, 2316 (1997).

${ }^{25}$ C.W.J. Beenakker and J.A. Melsen, Phys. Rev. B 50, 2450 (1994).

${ }^{26}$ J.M. van Ruitenbeek, in Mesoscopic Electron Transport, edited by L.L. Sohn, L.P. Kouwenhoven, and G. Schön (Kluwer, Dordrecht, 1997).

${ }^{27}$ P.W. Brouwer, S.A. van Langen, K.M. Frahm, M. Büttiker, and C.W.J. Beenakker, Phys. Rev. Lett. 79, 913 (1997); S.A. van Langen, P.G. Silvestrov, and C.W.J. Beenakker, Superlattices Microstruct. 23, 691 (1998); S.F. Godijn, S. Möller, H. Buhmann, L.W. Molenkamp, and S.A. van Langen, cond-mat/9811181 (unpublished).

${ }^{28}$ C. Untiedt, G. Rubio, S. Viera, and N. Agrait, Phys. Rev. B 56, 2154 (1997).

${ }^{29}$ H. Ohnishi, Y. Kondo, and K. Takayanagi, Nature (London) 395, 780 (1998); A.I. Yanson, G.Rubio Bollinger, H.E. van den Brom, N. Agrait, and J.M. van Ruitenbeek, ibid. 395, 783 (1998).

${ }^{30}$ B. Ludoph and J.M. van Ruitenbeek, Phys. Rev. B 59, 12290 (1999).

${ }^{31}$ J.A. Torres and J.J. Sáenz, Phys. Rev. Lett. 77, 2245 (1996).

${ }^{32}$ J.A. Torres J.I. Pascual, and J.J. Sáenz, Phys. Rev. B 49, 16581 (1994).

${ }^{33}$ F. Ott, S. Barberan, J.G. Lunney, J.M.D. Coey, P. Berthet, A.M. de Leon-Guevara, and A. Revcolevschi, Phys. Rev. B 58, 4656 (1998).

${ }^{34}$ C.A. Stafford, D. Baeriswyl, and J. Bürki, Phys. Rev. Lett. 79, 2863 (1997); J.M. van Ruitenbeek, M.H. Devoret, D. Esteve, and C. Urbina, Phys. Rev. B 56, 12566 (1997). 